DRAFT VERSION FEBRUARY 14, 2018

Preprint typeset using $\mathrm{LT}_{\mathrm{E}} \mathrm{X}$ style emulateapj v. 10/10/03

\title{
ANGULAR DIAMETER DISTANCE MEASUREMENT WITH GALAXY CLUSTERING IN THE MULTIPOLE SPACE
}

\author{
ASANTHA COORAY \\ Center for Cosmology, Department of Physics and Astronomy, University of California, Irvine, CA 92697 \\ Draft version February 14, 2018
}

\begin{abstract}
The shape of the angular power spectrum of galaxies in the linear regime is defined by the horizon size at the matter-radiation equality. When calibrated by cosmic microwave background measurements, the shape of the clustering spectrum can be used as a standard ruler to estimate angular diameter distance as a function of redshift at which galaxy clustering is measured. We apply the proposed cosmological test of Cooray et al. (2001) to a recent set of luminous red galaxy angular clustering spectra from the Sloan Digital Sky Survey between redshifts of 0.2 and 0.6 . Using the overall shape of the clustering power spectrum in the linear regime, we measure comoving angular diameter distances to eight redshift bins by marginalizing over the bias factors that determine the overall amplitude of the clustering spectrum in each of the bins. The Hubble constant consistent with these distance estimates is $68.5_{-6.1}^{+6.7} \mathrm{~km} \mathrm{~s}^{-1} \mathrm{Mpc}^{-1}$ at the $68 \%$ confidence level. We comment on the expected improvements with future surveys and the potential to measure dark energy parameters with this method.

Subject headings: cosmology: observations — cosmology: theory — galaxies: fundamental parameters — large scale structure
\end{abstract}

\section{INTRODUCTION}

The cosmic distance scale, either in terms of the luminosity using standard candles or the size using standard rulers, is one of the strongest probes of the cosmological expansion rate of the Universe and the dark energy responsible for recent acceleration (e.g., Huterer \& Turner 2001). It is well known that features in the clustering spectrum that are associated with known physical scales provide a basis to establish standard ruler techniques to measure distances between us and the redshift at which clustering is measured (Eisenstein et al. 1998). Using the sound horizon at the last scattering surface as the physical scale, this has been successfully applied to determine the distance to redshift $z \sim 10^{3}$ using the cosmic microwave background (CMB) anisotropy spectrum (Spergel et al. 2003; Spergel et al. 2006). The angular power spectrum of sources at low redshifts provides a similar test based on the standard ruler defined by the overall shape of the projected angular power spectrum (Cooray et al. 2001).

In the adiabatic cold dark matter model for structure formation, this standard ruler is the horizon at matter-radiation equality with the absolute physical scale directly calibrated through CMB anisotropy information. Similarly, another physical scale in the source power spectrum that is now well explored in the literature is the horizon at the end of Comptondrag that results in baryon oscillations (Blake \& Glazebrook 2003; Seo \& Eisenstein 2003). While the signature of baryon oscillation has now been detected in the galaxy correlation function at low redshifts with the Sloan Digital Sky Survey (SDSS; Eisenstein et al. 2005; also with 2dF Galaxy Redshift Survey by Cole et al. 2005), resulting in a 5\% measurement of the absolute distance to $z \sim 0.35$, this ruler has yet to be applied at multiple redshifts. On the other hand, adequate clustering measurements with wide-field surveys such as SDSS now exist to apply the standard ruler technique defined by the overall shape of the angular power spectrum to estimate distances out to several bins in redshift.

Since this standard ruler technique to measure distances is a purely geometric test using the linear power spectrum, it is insensitive to uncertainties on the exact shape of the galaxy power spectrum at non-linear scales. Issues related to non-linear clustering and corrections related to the conversion from real space to redshift space clustering are, however, concerns for distance measurements using baryon oscillations (Eisenstein et al. 2006). In galaxy clustering models based on the halo model (Cooray \& Sheth 2002) or extensions based on conditional luminosity functions (Cooray 2006) galaxy bias is scale independent at large physical scales corresponding to the linear regime. When estimating distances with angular power spectra the bias can be established concurrently, though, if bias is independently known one can separate the normalization of the power spectrum to obtain an extra handle on the cosmological parameters using the growth function of density fluctuations.

In this Letter we apply the proposed technique of Cooray et al. (2001) to a recent set of clustering measurements related to luminous red galaxies at redshifts between 0.2 and 0.6 from the Sloan Digital Sky Survey (Padmanabhan et al. 2006). The clustering measurements in the multipole space are divided to 8 bins in redshifts using photometric redshifts. We measure comoving angular diameter distance to each of these redshift bins and combine them to extract the Hubble parameter today. We also comment on the extent to which distance measurements can be improved in the future with this technique.

This Letter is organized as following: In the next Section, we will briefly outline the standard ruler technique involving the angular power spectrum of galaxies and a brief overview of clustering measurements used for the analysis. Section 3 describes results from our model fitting and a brief discussion of the extent to which this technique can be extended to measure dark energy parameters.

\section{DISTANCE MEASUREMENT}

We refer the reader to Cooray et al. (2001) for a detailed discussion related to this standard ruler method to measure distances. We briefly summarize the main points by first writing the angular power spectrum of galaxies in $i$ th redshift bin through the Limber (1954; Kaiser 1992) projection of the halo 


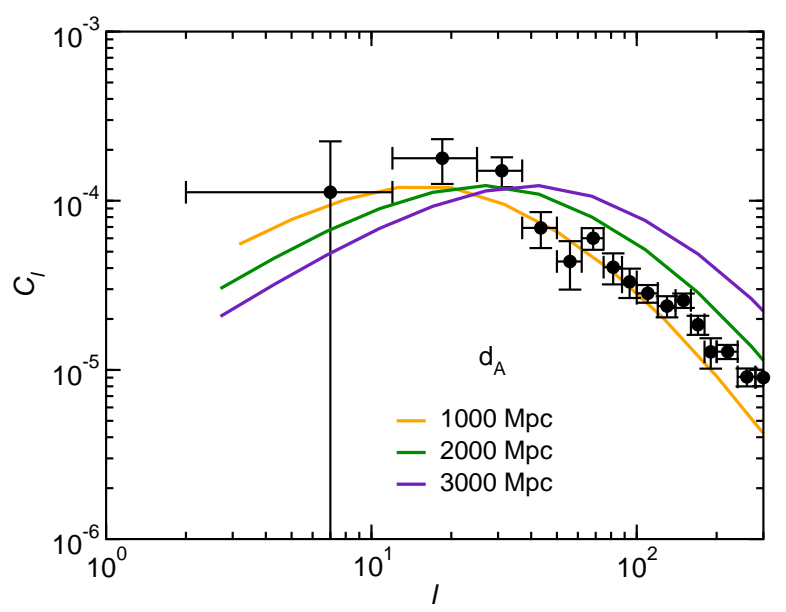

FIG. 1.- The standard ruler test illustrated using angular power spectrum of luminous red galaxies in the redshift bin 0.35 to 0.4 . The measurements are from Padmanabhan et al. (2006), while the solid lines show the predictions for clustering based on the linear power spectrum defined by $\Omega_{m} h^{2}=0.132$ and projected at several comoving angular diameter distances. In model fitting the data to extract distance, we marginalize over the overall normalization of the angular spectrum given by the parameter $F(z)$ (equation 4 ) and the uncertainty in $\Omega_{m} h^{2}$ from CMB measurements.

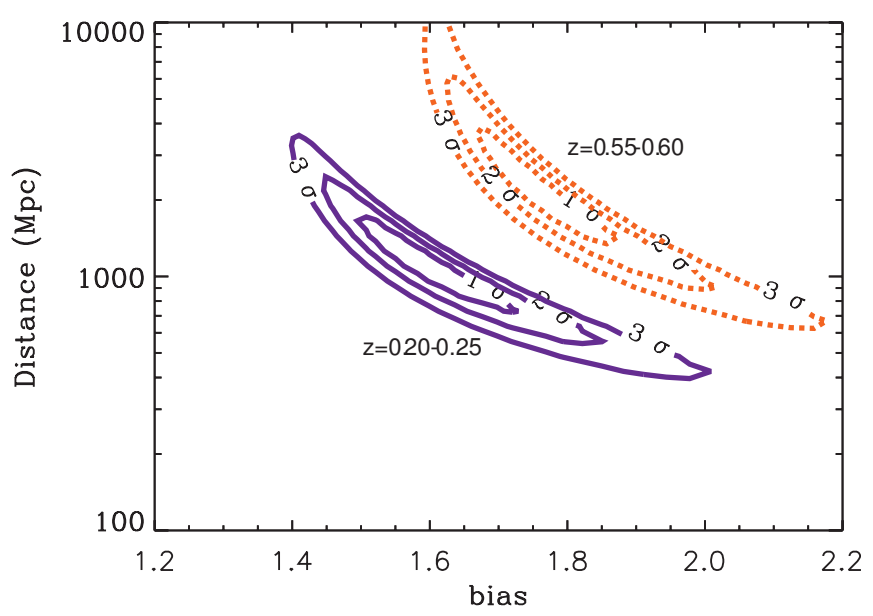

FIG. 2.- The constraints on parameters describing the galaxy bias and the comoving angular diameter distance for two redshift bins at $z=0.2$ to 0.25 and $z=0.55$ to 0.6 . Note that the two parameters considered for modeling in this paper is the distance and the parameter $F(z)$, though for easy comparison, we have plotted the bias factor by setting other parameters of $F(z)$ in equation 4 with values given by the recent concordance LCDM model consistent with the WMAP third-year analysis (Spergel et al. 2006).

number density power spectrum

$$
C_{l}^{i}=\int d z W_{i}^{2}(z) \frac{H(z)}{d_{A}^{2}(z)} P_{s s}\left(\frac{l}{d_{A}} ; z\right),
$$

where $W_{i}(z)$ is the normalized redshift distribution of galaxies in the given redshift bin so that $\int d z W_{i}(z)=1, H(z)$ is the Hubble parameter, and $d_{A}$ is the angular diameter distance in comoving coordinates ${ }^{1}$.

\footnotetext{
${ }^{1}$ Note that we use the comoving coordinates throughout here. There is an additional factor of $(1+z)$ involved between our definition of $d_{A}$ and the one commonly called angular size distance
} with

We assume that the sources trace the linear density field

$$
P_{s s}(k ; z)=b^{2}(z) D^{2}(z) P^{\operatorname{lin}}(k ; 0),
$$

where $b$ is the mass-averaged halo bias parameter, $P^{\text {lin }}(k ; 0)$ is the present day matter power spectrum computed in linear theory, and $D(z)$ is the linear growth function $\delta^{\operatorname{lin}}(k ; z)=$ $D(z) \delta^{\text {lin }}(k ; 0)$. Note that the halo model suggests a scaleindependent galaxy bias factor in the linear regime (e.g. Cooray \& Sheth 2002). Equation (1) then becomes

$$
\begin{aligned}
C_{l}^{i} & =\int d z W_{i}^{2}(z) F(z) P^{\operatorname{lin}}\left(\frac{l}{d_{A}^{i}} ; 0\right), \\
F(z) & =\frac{H(z)}{d_{A}^{2}(z)} D(z)^{2} b^{2}(z) .
\end{aligned}
$$

where the function $F_{i}(z)$, associated with redshift bin $i$, contains information on the halo bias, the growth function, the power spectrum normalization, and terms involved in the 3-D to 2-D projection (such as a $1 / d_{A}^{2}$ term; see, Eq. (1).

The angular diameter distance measurement involves following standard rulers: the horizon at matter radiation equality, $k_{\text {eq }}=\sqrt{2 \Omega_{m} H_{0}^{2}\left(1+z_{\text {eq }}\right)} \propto \Omega_{m} h^{2}$, which controls the overall shape of the power spectrum, and the sound horizon at the end of the Compton drag epoch, $k_{\mathrm{s}}\left(\Omega_{m} h^{2}, \Omega_{b} h^{2}\right)$, which determines baryon acoustic oscillations in the power spectrum. The angular or multipole locations of these features shift in redshift as $l_{\mathrm{eq}, \mathrm{s}}=k_{\mathrm{eq}, \mathrm{s}} d_{A}\left(z_{i}\right)$. Thus, the test involves measuring $C_{l}^{i}$ in several redshift bins and using the fact that $l_{\mathrm{eq}}$ scales with $d_{A}\left(z_{i}\right)$ to constrain the angular diameter distance as a function of redshift. Here, to be conservative, we ignore information at non-linear scales and only use measurements in the linear regime with $l<200$. We also do not pursue a test involving the baryon features since these are not detected at a reasonable significance in each of the bins, though, when clustering measurements in all bins are combined, there is a 2.5 sigma evidence for the presence of oscillations (Padmanabhan et al. 2006).

Note that in our analysis we use clustering measurements in bins $\Delta z$ of 0.05 . With such small bins in redshift, we found the exact shape of $W_{i}(z)$ to only lead to few percent corrections to the distance. These distributions, however, are established with photometric redshift measurements for each of the galaxies in Padmanabhan et al. (2006). For surveys where exact distributions are less certain, the uncertainty in $W_{i}(z)$ can be marginalized over when estimating distances.

The standard ruler test assumes that the Limber approximation is adequate for a reliable distance measurement with galaxy clustering angular power spectrum. We have numerically tested the accuracy of the approximation by integrating the exact formula for $C_{l}$ and have found the Limber approximation is accurate to better than $0.2 \%$ for redshift bins used in the present analysis. With small redshift bins, there is one complication here involving redshift space distortions associated with peculiar velocities. Using the Kaiser (1987) formula related to enhancement of power at large angular scales due to bulk motions, we included terms to related to velocityvelocity and velocity-density corrections to the galaxy clustering spectrum (also, see, discussion in Padmanabhan et al. 2006). These terms mostly affect the overall normalization and the determination of galaxy bias parameters and is only responsible for less than a $2 \%$ systematic change to the distance. 

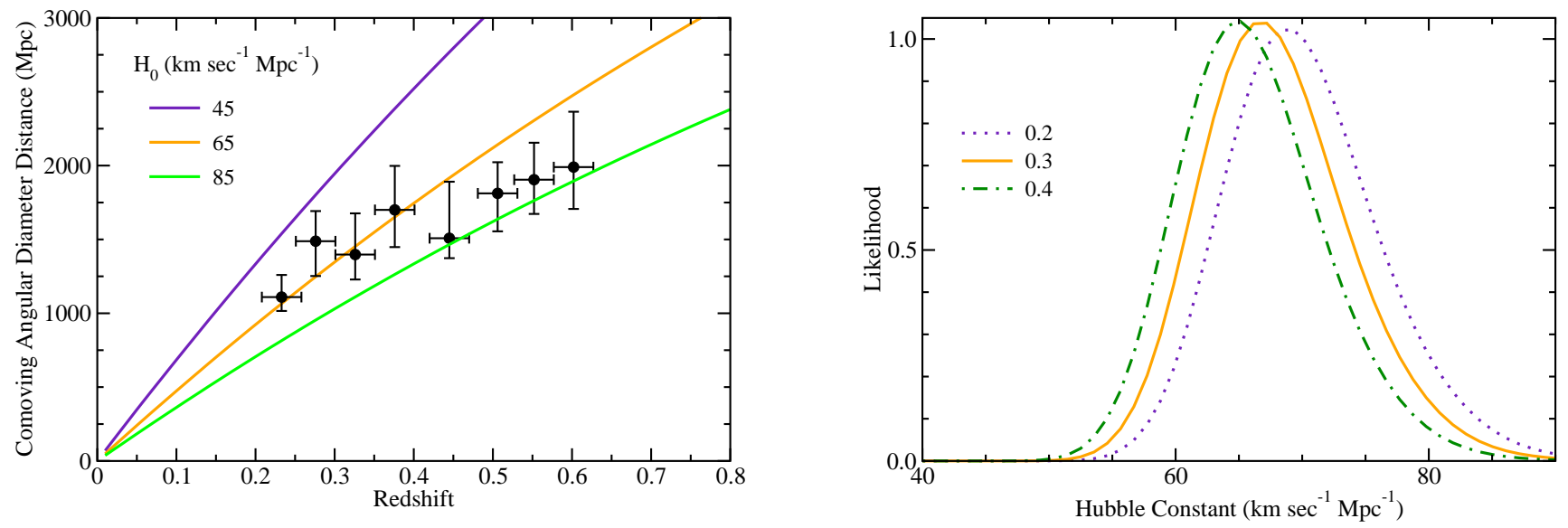

FIG. 3. - Left: The comoving angular diameter distance as a function of redshift for the eight bins considered by Padmanabhan et al. (2006) for clustering measurements. The distance estimates are obtained by marginalizing over the normalization parameter $F(z)$. For reference, we show three difference estimates on the comoving distance based on the Hubble constant today assuming a flat- $\Lambda$ CDM cosmology with $\Omega_{m}=0.27$. Right: The likelihood distribution of the Hubble constant as a function of three assumed values for $\Omega_{m}$. For $\Omega_{m}=0.2, H_{0}=68.5_{-6.1}^{+6.7}$ at the $68 \%$ confidence level.

\section{RESULTS AND DISCUSSION}

Though the technique is clear, what has been lacking so far are adequate clustering measurements that span large angular scales, or small multipoles, so that the overall shape of the angular power spectrum of galaxies is determined at several redshifts. The recent measurements of galaxy clustering in the angular multipole space by Padmanabhan et al. (2006) provide a first set of data to apply the proposed technique to estimate distances. The measurements involve the luminous red galaxies in the Sloan Digital Sky Survey at estimated photometric redshifts between 0.2 and 0.6. The measurements are subdivided to eight redshift bins of width 0.05 .

In Padmanabhan et al. (2006), the clustering measurements in each of the redshift bins and the cross-clustering between redshift bins were used to estimate the three-dimensional power spectrum of galaxies through an inversion from two dimensional $C_{l}$ to three-dimensional $P(k)$. Since no attempt was made to extract individual distances there, other than to quantify the extent to which a mean distance can be measured for the whole sample, we carry out an explicit analysis to estimate absolute distances to each of the bins following the proposed technique of Cooray et al. (2001). Figure 1 summarizes the underlying concept behind the proposed method using clustering measurements in one of the redshift bins as an example. We refer the reader to Padmanabhan et al. (2006) for details on the clustering measurements as well as a figure of all power spectra in the eight redshift bins.

In addition to distance $d_{i}(z)$ we also estimate the parameter $F_{i}(z)$ to each of the redshift bin $i$. The underlying power spectrum is taken to be the one consistent with WMAP third year analysis given in Spergel et al. (2006; WMAP+SDSS). The overall normalization of the matter power spectrum is not an important parameter for this analysis since it's uncertainty can also be included with $F_{i}(z)$. The main parameter of importance is $\Omega_{m} h^{2}$ and we marginalize over the WMAP uncertainty by assuming that the likelihood is a Gaussian. In future, a more thorough analysis could jointly fit CMB data and angular power spectra in each of the bins at the same time though we do not pursue such an analysis due to fractionally larger uncertainties in the galaxy clustering spectra than the CMB clustering spectrum.

In Figure 2, we highlight the constraints on the distances to two redshift bins. For illustrative purposes and easy comparison with Padmanabhan et al. (2006) results, instead of the normalization parameter $F_{i}(z)$, using the $\Lambda$ CDM concordance cosmology, we show the bias factor $b_{i}(z)$, consistent with each of the estimated $F_{i}(z)$. The parameter fitting makes use of the full covariance matrix of the clustering measurements. Our estimates for bias factors are generally consistent with those given in Padmanabhan et al. (2006) with small differences arising due to small variations in the concordance parameters considered when estimating $F(z)$ and the description of the peculiar velocity spectrum related to redshift space distortion corrections.

In Figure 3 left panel, we marginalize over $F_{i}(z)$ and plot $d_{i}(z)$ as a function of redshift to make a Hubble diagram of comoving angular diameter distances at multiple redshifts using the standard ruler captured by the angular clustering spectrum of galaxies. To each of the redshift bins, we measure distances with a typical accuracy of $\sim 10 \%$ to $20 \%$. In Figure 3 right panel, we fit the distance estimates to extract the Hubble constant today. We only fit a single parameter to the Hubble diagram as we found out that there is no significant information related to, say, dark energy parameters with distances measured to the accuracies found here. The implied Hubble constant is $68.5_{-6.1}^{+6.7} \mathrm{~km} \mathrm{~s}^{-1} \mathrm{Mpc}^{-1}$, at the $68 \%$ confidence level. This value is consistent with recent estimates of the Hubble constant based on WMAP and large-scale structure power spectrum (Spergel et al. 2006) as well as independent measurements involving the Sunyaev-Zel'dovich effect (Bonamente et al. 2005).

The uncertainty in the estimated Hubble constant is slightly larger than the $\sim 6 \%$ accuracy of mean distance to a mid redshift of the range suggested in Padmanabhan et al. (2006; see their Section 5.1). The difference is partly due to our use of only the overall shape for the standard ruler method while the suggestion in Padmanabhan et al. (2006) also makes use of 
the implied overall $2.5 \sigma$ detection of baryon oscillations in the combined power spectrum from angular spectra in each of the 8 redshift bins. The accuracy of $\sim 10 \%$ for the Hubble constant we find here, however, is in good agreement with the suggestion in Padmanabhan et al. (2006) that, with a negligible baryon fraction, a mean distance to $z \sim 0.5$ can be established with an accuracy of $10 \%$ using the combined clustering power spectrum. Here, we have explicitly carried out the necessary analysis and have established a Hubble diagram using clustering measurements for the first time using a standard ruler technique.

While the distances are estimated roughly to fractional accuracies between $10 \%$ to $20 \%$ in most redshift bins, for precision dark energy measurements, we find that distance estimates with accuracies below a few percent are required. Using the overall shape of the clustering spectrum alone, such measurements are challenging, though not impossible. The clustering measurements used for the present analysis involve on average 40,000 to 60,000 galaxies in each of the redshift bins over a total imaging area of 3500 square degrees. For $5 \%$ distance measurements in 0.05 redshift bins, the sample sizes of galaxies in each of the redshift bins must be increased by a factor more than 4 . This could be achieved by increasing the sky survey area to roughly a half of the sky for galaxy samples out to a redshift of 0.5 or so. Though this is a large requirement, such data will be available with expected catalogs from survey instruments such as the Large Synoptic Survey Telescope (LSST; Zhan et al. 2006).

Similarly, one needs to push these measurements beyond redshift of 0.6 now probed with SDSS. At high redshifts, the area requirement becomes smaller as the volume is increased. We find that adequate distance measurements out to $z \sim 1$ to 1.5 can be achieved with surveys of a few thousand square degrees provided that galaxy samples are still selected to be those involving large bias factors so the clustering amplitude is large. The proposed Dark Energy Survey (DES;
Annis et al. 2005) will provide a first dataset to measure distances with this standard ruler technique out to redshifts of 1 and slightly larger. At redshifts between 2 and 4, a very large area ( $>100$ square degrees) Lyman-break galaxy survey could provide adequate measurements for $10 \%$ accurate absolute distances to such redshifts.

In any case, it is unlikely that precision dark energy measurements can be achieved with distances measured from the overall clustering shape alone. A factor of 4 to 5 improvement in distance measurements could be easily achieved with precision detection of baryon oscillations since this feature is sharper than the physical ruler related to the horizon at matterradiation equality. Such measurements, however, must overcome uncertainties and systematics related to non-linear clustering though most concerns have now shown to lead to at most a few percent systematic corrections to, say, the location of the baryon peak (Eisenstein et al. 2006). The angular diameter distances from galaxy clustering may be more useful in combination with other cosmological distance measurements, such as luminosity distances from type Ia supernovae since the combination breaks degeneracies between certain cosmological parameters. Furthermore, is certain non-standard cosmologies (e.g., Uzan et al. 2004), the luminosity and angular diameter distances may not follow the usual cosmological relation of $d_{L}=d_{A}(1+z)^{2}$. Thus, in addition to large samples of supernovae, techniques to improve angular diameter distances measurements must also be pursued in the future for a variety of purposes. Thus, it is likely that the standard ruler method based on features in the power spectrum, either the overall shape, baryon oscillation or both, will remain to be useful.

We thank Nikhil Padmanabhan for making available to us electronic data files of clustering measurements from Padmanabhan et al. (2006). This work was completed during author's stay at the Aspen Center for Physics.
Annis, J. et al. astro-ph/0510195

Blake, C. \& Glazebrook, K. 2003, ApJ, 594, 665

Cole, S. et al. 2005, MNRAS, 362, 505

Cooray, A., Hu, W., Huterer, D. \& Joffre, M. 2001, ApJ, 557, L7

Cooray, A. 2006, MNRAS, 365, 842

Cooray, A. \& Sheth, R. 2002, Physics Reports, 372, 1

Eisenstein, D.J., Hu, W., Tegmark, M. 1998, ApJ, 504, L57.

Eisenstein, D.J. et al. 2005, ApJ, 633, 560

Eisenstein, D.J., Seo, H.-J., White, M. 2006, astro-ph/0604361

Huterer, D. \& Turner, M. S. 2001, PRD, 64, 123527

\section{REFERENCES}

Kaiser, N. 1992, ApJ, 388, 286

Limber, D. 1954, ApJ, 119, 655

Padmanabhan, N. et al. 2006, MNRAS submitted iastro-ph/0605302

Seo, H.-J. \& Eisenstein, D. J. 2003, ApJ, 598, 720

Spergel, D. N. et al. 2003, ApJS, 148, 175

Spergel, D. N. et al. 2006, astro-ph/0603449

Uzan, J.-P., Aghanim, N., Mellier, Y. 2004, PRD, 70, 083533

Zhan, H., Knox, L., Tyson, J. A. \& Margoniner, V. 2006, ApJ, 640, 8 